\title{
Xiayuxue decoction reduces renal injury by promoting macrophage apoptosis in hepatic cirrhotic rats
}

\author{
C. Liu ${ }^{1 *}$, J. Cai ${ }^{2 *}$, Z. Cheng ${ }^{1}$, X. Dai ${ }^{1}$, L. Tao ${ }^{3}$, J. Zhang ${ }^{3}$ and D. Xue ${ }^{3}$ \\ 'Laboratory of Molecular Pathology, Central Laboratory, Putuo Hospital, \\ Shanghai University of Traditional Chinese Medicine, Shanghai, China \\ 2Department of General Surgery, Putuo Hospital, \\ Shanghai University of Traditional Chinese Medicine, Shanghai, China \\ ${ }^{3}$ Department of Infectious Disease, Putuo Hospital, \\ Shanghai University of Traditional Chinese Medicine, Shanghai, China \\ *These authors contributed equally to this study. \\ Corresponding authors: D. Xue / J. Zhang \\ E-mail: dongying11@citiz.net / pzxgrk@163.com \\ Genet. Mol. Res. 14 (3): 10760-10773 (2015) \\ Received February 4, 2015 \\ Accepted July 10, 2015 \\ Published September 9, 2015 \\ DOI http://dx.doi.org/10.4238/2015.September.9.15
}

ABSTRACT. Renal pathological changes in cirrhotic rat have not been extensively reported. The aim of this study was to investigate whether Xiayuxue decoction (XYXD) could attenuate renal injury induced by bile duct ligation (BDL), with special focus on the mechanisms promoting renal macrophage apoptosis. The rats were treated with BDL for 5 weeks and administered $0.36 \mathrm{~g} / \mathrm{kg}$ XYXD intragastrically from day 1 of initiating BDL. Renal tissue was monitored by hematoxylin-eosin and Sirius red staining. Macrophage infiltration and proinflammatory cytokines such as tumor necrosis factor and chemokine ligand 2 were detected by quantitative polymerase chain reaction. Macrophage apoptosis was detected by double immunofluorescence staining. Blood urea nitrogen, creatinine, and glomerulus diameter increased significantly after a 5-week BDL treatment in XYXD (BDL-XYXD) rats. CD68 and pro-inflammatory cytokine 
mRNA increased in the kidneys of control (BDL-water) rats. Fluorescence microscopy analysis showed that $X Y X D$ promoted apoptosis in renal CD68+ macrophages. Collogen1 (Col 1), pro-fibrogenic cytokines, and a-smooth muscle actin in kidneys of BDL-water rats increased significantly compared to the sham group. XYXD inhibited Col 1 and pro-fibrotic factors in BDL-XYXD rats. Our results demonstrated that XYXD significantly reduced renal injury by, at least in part, promoting macrophage apoptosis in rats with damaged renal histopathology due to BDL-induced cirrhosis.

Key words: Macrophage; Apoptosis; Renal injury; Xiayuxue decoction; Liver cirrhosis

\section{INTRODUCTION}

Liver fibrosis and cirrhosis, the common end stage of most chronic liver diseases (Chiu et al., 2014; Hong et al., 2014), is triggered by chronic liver injury caused by various etiologies including viral infection, cholestasis, metabolic diseases, and alcohol abuse (Patsenker et al., 2009). Liver cirrhosis is a systematic disease where organs such as the spleen and kidneys are also involved (Arroyo, 2013). The pathology of spleen and portal hypertension has been well studied in liver cirrhosis (Mejias et al., 2009). Clinical evidence indicates that the kidney is also injured in cirrhotic patients (Wong et al., 2008; Mejias et al., 2009; Belcher et al., 2014). However, renal pathology in liver cirrhosis has not been extensively studied. Therefore, studies on the pathophysiology of the kidney in liver cirrhosis would be of value.

Macrophages have a broad repertoire of context-dependent, immune, inflammatory and regulatory functions in fibrosis and cirrhosis (Heinrichs et al., 2011; Heymann et al., 2012; Andersen et al., 2014). We have previously shown that Kupffer cells (hepatic macrophages) underwent apoptosis and might exert fibrogenic function by producing collogen 1 (Col 1) independently during liver fibrosis (Liu et al., 2010). However, renal macrophage function in liver cirrhosis has been rarely reported and requires urgent investigation.

Xiayuxue decoction (XYXD) was first described in Shanghan Za Bing Lun's Synopsis of the Golden Chamber in 200 AD. It consists of 3 medicinal herbs mixed in specific proportions as follows: Radix et Rhizoma Rhei (10 g), Semen Persicae (10 g), and Eupolyphaga Seu Steleophaga (6 g). XYXD has been used for the treatment of puerpera-related abdominal pain and oligomenorrhea caused by blood stasis for more than 1800 years without side effects. XYXD has also widely used to treat liver fibrosis and cirrhosis in China (Chen, 2013) and is reported to suppress liver fibrosis/ cirrhosis induced by carbon tetrachloride (CCL4) (Zhang et al., 2014), thioacetamide (TAA) (Ding et al., 2012), and pig serum (Chen et al., 2012). XYXD has been reported to exert a protective effect in diabetic nephropathy (Du et al., 2010). However, information is scarce regarding the protective action of XYXD on the kidney in a cirrhotic model.

Therefore, in this study, we first investigated the potential antifibrogenic effects of XYXD in a bile duct ligation (BDL) model. Second, we ascertained whether XYXD ameliorates renal damage during cirrhosis as well as cirrhosis-induced nephropathology, with special focus on the mechanisms inhibiting activation and promoting apoptosis of renal macrophages. These findings help our understanding of important mechanisms involving renal macrophages during cirrhosis. 


\section{MATERIAL AND METHODS}

\section{Reagents}

ApopTag Fluorescein In Situ Apoptosis Detection Kit (S7110) was purchased from Chemicon (Temecula, CA, USA). Anti-a- smooth muscle actin (SMA) mouse antibody was obtained from Sigma (Saint Louis, MO, USA) and diluted 1:400. Anti-Fas mouse antibody was purchased from BD Pharmingen (San Jose, CA, USA) and diluted 1:1000. Anti-CD68 mouse antibody was purchased from Serotec (Oxford, OX5, UK) and diluted 1:100. Anti-Bax rabbit antibody was purchased from CST (Beverly, MA, USA) and diluted 1:100. Anti-pSmad2/3 rabbit antibody was obtained from Santa Cruz (Dallas, TX, USA) and used in 1:100 dilution.

\section{Preparation of XYXD}

XYXD consists of crude slices from 3 medicinal substances in the following ratios: Radix et Rhizoma Rhei (10 g), Semen Persicae (10 g), and Eupolyphaga Seu Steleophaga (6 g). The medicines were accredited by a pharmacologist. The medicinal mixture was extracted twice using $75 \%$ ethanol and filtered, the resulting extract was evaporated and dehydrated under vacuum. The extract powder was weighed and used by dissolving in pure water at the desired concentrations.

\section{Animals}

Male Wistar rats received humane care with unlimited accesses to chow food and water during the study. All study protocols complied with ethical considerations of the Animal Ethic Committee at Shanghai University of Traditional Chinese Medicine, which is in accordance with the National Research Council criteria. All animal experiments and procedures were reviewed and approved by the Institutional Animal Care and Use Committee of Shanghai University of Traditional Chinese Medicine and were performed in accordance with the relevant guidelines and regulations.

\section{BDL-induced cirrhosis model}

Rats were divided into 3 groups. The cirrhotic model group underwent BDL, as previously described (Francis et al., 2007; Graf et al., 2014) and was administered distilled water (BDL-water). The XYXD treatment group underwent BDL and was administered XYXD intragastrically daily for 5 weeks (BDL-XYXD), at a dose of $0.36 \mathrm{~g} / \mathrm{kg}$ body weight, which is equivalent to human doses in clinical therapeutics. The sham group received distilled water orally (sham). All rats were sacrificed under $2 \%$ sodium pentobarbital anesthesia while making all efforts to minimize suffering, and kidney as well as liver samples were collected for analysis.

\section{Liver and renal histopathology}

Liver and kidneys were fixed in $10 \%$ paraformaldehyde, embedded in paraffin, sectioned, and stained with hematoxylin and eosin (H\&E) and Sirius red. The glomerular 
diameter was measured and Sirius red-stained collagen was quantitated in 6 randomly-selected fields of view per sample. The glomerular diameter and area of Sirius red-positive staining was quantified using a computer-aided image analysis software Image-Pro Plus version 6.1 (MediaCybernetics, USA).

\section{Immunohistochemistry}

After deparaffinization and dehydration, microwave antigen retrieval was performed for 5 min in sodium citrate buffer, after which the sections were treated with $0.6 \% \mathrm{H}_{2} \mathrm{O}_{2}$ for 10 min. Sections were then blocked with $5 \%$ bovine serum albumin (BSA) for 30 min and incubated with primary antibody (anti-a-SMA). After washing in phosphate-buffered saline (PBS) twice, sections were incubated with biotinylated secondary antibody for $30 \mathrm{~min}$.

\section{Western blotting}

The tissues were lysed in a buffer containing $40 \mathrm{mM}$ Tris-HCl, pH 7.5, $150 \mathrm{mM} \mathrm{NaCl}, 2 \mathrm{mM}$ EDTA, $1 \mathrm{mM}$ DTT, $1 \%$ Triton X-100, $2 \mathrm{mM} \mathrm{Na}_{3} \mathrm{VO}_{4}, 10 \mathrm{mM} \mathrm{NaF}$, and $10 \mathrm{mM}$ sodium pyrophosphate supplemented with a protease inhibitor cocktail mixture (Sigma). Protein content was measured with a bicinchoninic acid assay kit (Jiancheng Institute of Biotechnology, Nanjing, China). Samples were separated by sodium dodecyl sulfate-polyacrylamide gel electrophoresis (SDS-PAGE) gel electrophoresis and proteins were transferred onto an Immobilon-P membrane. After a blocking procedure, the membrane was incubated overnight with anti-transforming growth factor (TGF)- $\beta 1$, p-Smad $2 / 3$ or anti-GAPDH antibody at $4^{\circ} \mathrm{C}$, followed by incubation with a secondary antibody coupled to horseradish peroxidase.

\section{Real-time reverse-transcriptase (RT)-quantitative polymerase chain reaction (qPCR)}

RNA was extracted from whole liver tissue, using RNA extraction kits (Qiagen), according to the manufacturer instructions. First-strand cDNAs were synthesized using SuperScript VILO kit (Invitrogen, Carlsbad, CA, USA). qPCR was performed using SYBR Green for 40 cycles of $15 \mathrm{~s}$ at $95^{\circ} \mathrm{C}$ and $60 \mathrm{~s}$ at $60^{\circ} \mathrm{C}$ using a Rotor-Gene 6000 system according to the manufacturer instructions. Sequences and accession numbers of the primers used in real-time PCR were as follows: CD68: 5'-TGTACCTGACCCAGGGTGGAA-3' (forward primer) and 5'-GAATCCAAAGGTAAGCTGTCCG TAA-3' (reverse primer); TGF- $\beta 1$ : 5'-GCCTGAGTGGCTGTCTTTTG-3' (forward primer) and 5'-ACCTCGACGTTTGGGACTG-3' (reverse primer); CCL2: 5'-ACCAGCCAACTCTCACTGAA-3' (forward primer) and 5'-GCCAGTGAATGAGTAGCAGC-3' (reverse primer); TNF- $\alpha$ : 5'GGCAGCCTTGTCCCTTGAAGAG-3' (forward primer) and 5'-GTAGCCCACGTCGTAGCAAACC -3' (reverse primer); A-SMA: 5'-CGAGAGGACGTTGTTAGCATAGAG-3' (forward primer) and 5'-GGGCATCCACGAAACCA-3' (reverse primer); Col1 (a1): 5'-ACTCAGCCGTCTGTGCCTCA -3' (forward primer) and 5'-GGAGGCCTCGGTGGACATTA-3' (reverse primer); $18 \mathrm{~S}$ rRNA: 5'-GTAACCCGTTGAACCCCATT-3' (forward primer) and 5'-CCATCCAATCGGTAGTAGCG-3' (reverse primer).

Results were analyzed by double $\Delta \mathrm{Ct}$ method. Values are reported as fold-change in comparison with control. All reactions were performed in triplicate. 


\section{Immunofluorescence staining}

To detect macrophage apoptosis, terminal deoxynucleotidyl transferase-mediated dUTP nick-end-labeling (TUNEL) assay was performed using a commercially available kit (S7110, Millipore) following the manufacturer instructions. Thereafter, the slides were incubated with $5 \%$ BSA for 30 min followed by incubation with primary antibody CD68 at $37^{\circ} \mathrm{C}$ for $1 \mathrm{~h}$. Slides were then washed thrice with PBS and incubated with the secondary Cy3-conjugated goat antimouse secondary antibody for $1 \mathrm{~h}$ at room temperature. Finally, the slides were fixed in mounting medium. Images were captured using an Olympus BX43 microscope (Olympus, Tokyo, Japan) and processed using the DP73-TWAIN version software.

\section{Statistical analysis}

We first examined the normality of the data. The results are reported as means \pm standard deviation (SD). For group comparisons, if the variances across the variables were equal, one-way analysis of variance LSD test was applied. The analyses were performed using SPSS version 18.0 statistical package (SPSS Inc., Chicago, IL, USA). If the variables were unequal using the nonparametric Kruskal-Wallis statistic Dunn's multiple comparison test, the analyses were performed with GraphPad Prism 5.0 (GraphPad Software, Inc., La Jolla, CA, USA). A P value $<0.05$ was considered to be statistically significant.

\section{RESULTS}

\section{XYXD inhibits hepatic collagen deposition in liver cirrhosis}

Liver cirrhosis was confirmed by several lines of evidence. First, rats with BDL for 5 weeks displayed marked bile duct proliferation with periductular collagen accumulation and incipient disruption of the lobular liver architecture (Figure 1A). BDL-XYXD group showed an improvement in histological parameters. The accumulation of extracellular matrix (ECM), especially collagen, was clearly revealed by Sirius red staining. As shown in Figure 1B, collagen staining was scarcely observed in the sham liver samples except in the area around central venous walls. Cirrhotic nodule formation was also seen in BDL-water samples. After 5-week

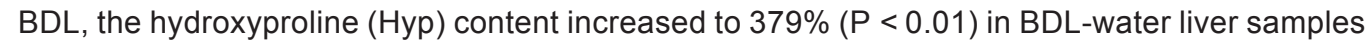
when compared with the sham. BDL-XYXD group showed a significantly $(P<0.05)$ reduced liver Hyp content when compared with that in BDL-water group. As sustained deposition of ECM mainly results from activation of hepatic stellate cells (HSCs), a correlation between accumulated collagen and activated HSCs was studied via immunohistochemistry to detect a marker for activated fibroblasts, $\alpha$-SMA in liver sections (Figure 1D). The expression of a-SMA and TGF- $\beta 1$ increased significantly in BDL-induced liver cirrhosis. There was remarkable difference in $\alpha-S M A$ and TGF- $\beta 1$ expression in the BDL-XYXD rats compared with BDL-water rats (Figure $1 \mathrm{E}$ and $\mathrm{F}$ ).

Taken together, these results confirm that BDL-treatment causes HSC activation and accumulation of ECM in liver cirrhosis. XYXD have a significantly inhibitory effect on hepatic collagen content in BDL-induced livers cirrhosis. 
Sham

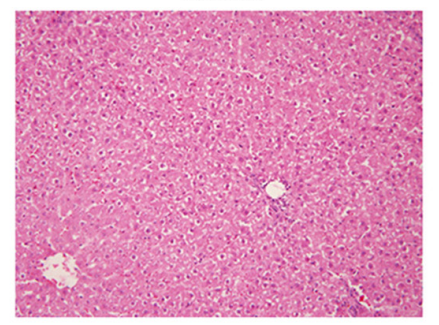

B

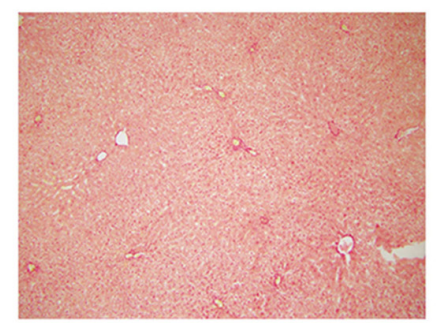

C
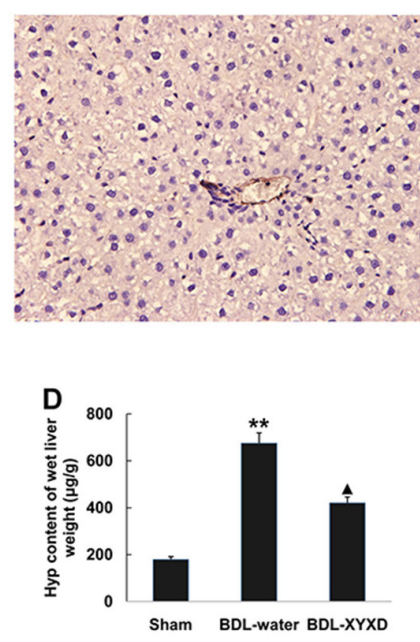

BDL-water
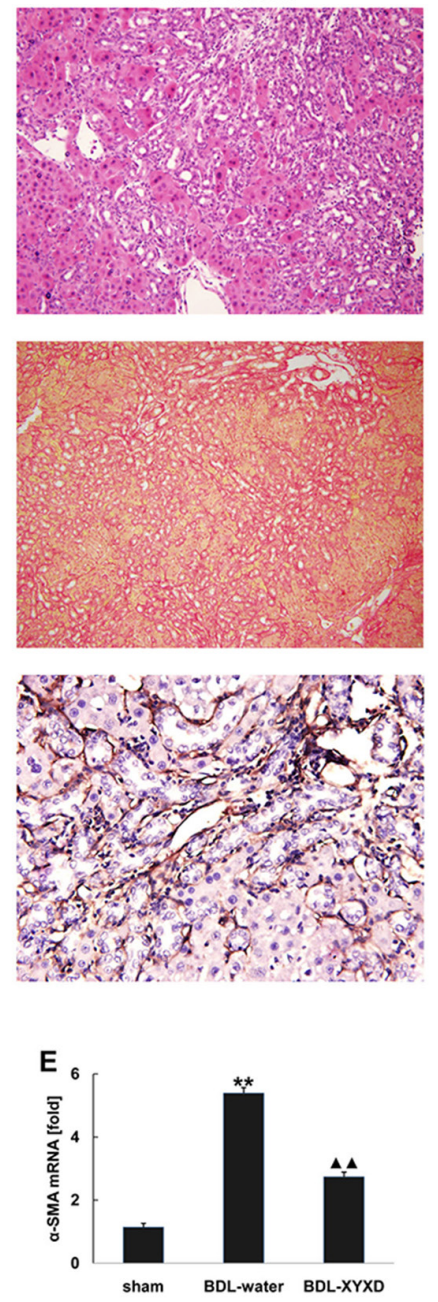

BDL-XYXD
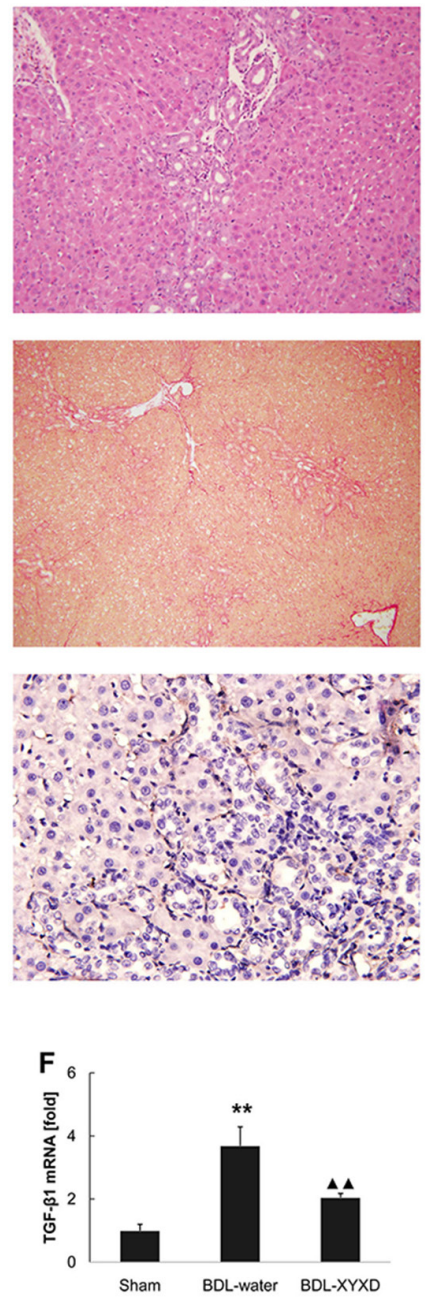

Figure 1. Effects of XYXD on histological changes in BDL-treated rat liver. A. Liver sections stained with H\&E (200X). B. Liver sections stained with Sirius red. C. Liver sections stained with a-SMA. D. Hydroxyproline content of liver tissue. E. Expression of $\alpha-S M A$ using real-time PCR $(N=4)$. F. Expression of TGF- $\beta 1$ using real-time PCR $(N=4)$. The data are reported as means $\pm \mathrm{SD}$. ${ }^{* *} \mathrm{P}<0.01$, vs sham; ${ }^{\Delta} \mathrm{P}<0.05,{ }^{\Delta}{ }^{\Delta} \mathrm{P}<0.01$ vs $\mathrm{BDL}$-water.

\section{Kidney injury in rats following BDL-treatment}

It is well documented that BDL induces liver cirrhosis. However, reports are scarce, regarding renal damage during $B D L$-induced cirrhosis in rats. Body weight of the rats from the BDL-water group was significantly lower than that of the sham group (Table 1$)(P<0.01)$. The kidney/body weight ratio was significantly higher in the BDL-water rats than that in the sham and BDL-XYXZ groups $(P<0.05)$, indicating that the kidney was damaged during BDL-induced liver cirrhosis. Renal function parameters deteriorated in rats subjected to BDL (Table 1). In BDL-water 
rats, BUN and creatinine levels were significantly higher compared with the sham rats $(P<0.01)$, indicating renal injury as well. Additionally, these BDL-induced higher levels were reduced with oral administration of XYXD.

Table 1. Organ weights and parameters of renal injury in rats with BDL-induced hepatic fibrosis.
\begin{tabular}{lccccc}
\hline Group (N) & Body weight (g) & Kidney weight $(\mathrm{g})$ & Kidney/body $(\%)$ & BUN $(\mathrm{mM})$ & $\operatorname{Cr}(\mu \mathrm{M})$ \\
\hline Sham (8) & $358.40 \pm 20.93^{\star}$ & $2.00 \pm 0.13$ & $5.60 \pm 0.50^{\star}$ & $8.30 \pm 0.97^{\star \Lambda}$ & $24.76 \pm 3.39^{\star}$ \\
BDL-water (7) & $304.73 \pm 34.20$ & $1.98 \pm 0.30$ & $6.53 \pm 0.59$ & $17.43 \pm 4.39$ & $66.86 \pm 7.99$ \\
BDL-XYXD (8) & $315.76 \pm 28.16$ & $1.87 \pm 0.14^{\star}$ & $5.96 \pm 0.81^{\star}$ & $13.13 \pm 2.41^{\star}$ & $46.25 \pm 6.73^{\star}$ \\
\hline
\end{tabular}

$\Delta \mathrm{P}<0.05$ and $\Delta \Delta \mathrm{P}<0.01$, versus BDL-water rats.

\section{Renal pathological changes in BDL-induced cirrhotic rats}

H\&E staining showed that inflammatory cells infiltrated in the space between tubules in BDL-water rats (Figure 2A). The glomerular diameter was significantly increased in BDL-water rats compared with that in sham (Figure $2 \mathrm{~B}$ ). There was marked reduction of inflammatory infiltration and glomerular diameter in the BDL-XYXD group. The inhibitory effect of XYXD was further confirmed by Sirius red staining (Figure $2 \mathrm{C}$ ), which stains cross-linked collagen in kidney sections. The amount of collagen deposition was decreased in the BDL-XYXD group. The deposition of collagen observed in the BDL-water group was significantly disrupted by XYXD (Figure 2D). Taken together, these results confirm renal damage following 5-week BDL-treatment.

A

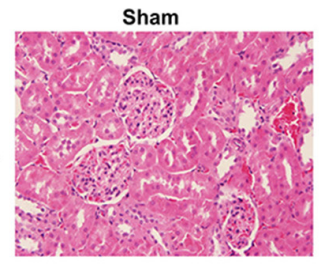

C

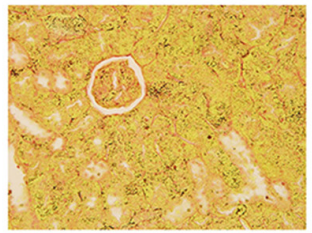

BDL-water
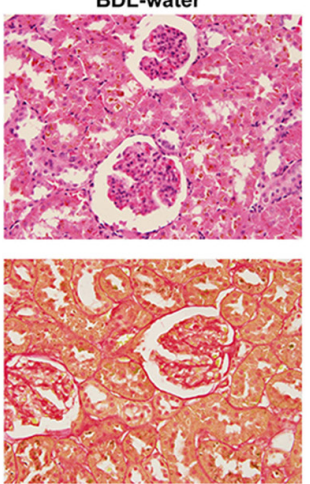

BDL-XYXD

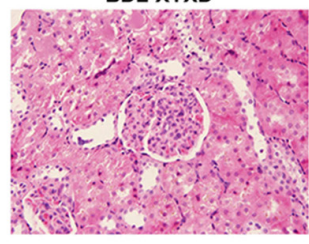

- II

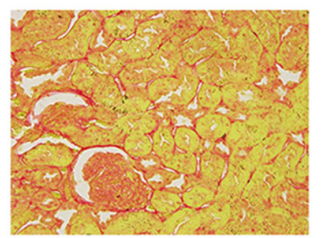

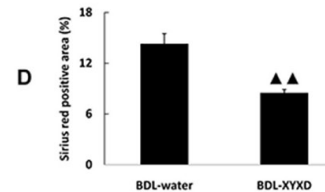

Figure 2. Effects of XYXD on renal histological changes in BDL-cirrhotic rats. A. Kidney sections stained with H\&E (400X). B. Glomerular diameter of kidney. C. Kidneys sections stained with Sirius red (400X). D. Quantitative results of Sirius red staining. $(\mathrm{N}=6)$. The data are reported as means $\pm \mathrm{SD}$. ${ }^{*} \mathrm{P}<0.05,{ }^{*} \mathrm{P}<0.01$, vs sham; ${ }^{\Delta} \mathrm{P}<0.05,{ }^{\Delta \Delta} \mathrm{P}$ $<0.01$ vs BDL-water.

\section{Macrophage activation in BDL-treated rats}

Macrophages play a key role in renal injury, therefore, a specific macrophage marker, CD68 was used to elucidate the function of renal macrophages. CD68+ macrophages were presented in glomeruli and were at very low levels in sham rats (Figure 3A). After BDL treatment for 5 weeks, CD68+ macrophages with strong staining appeared not only in glomeruli, but also between renal tubules. XYXD inhibited CD68-positive macrophage expression (Figure $3 A$ and $B$ ). 
Consistent with the results of immunostaining of CD68, real-time PCR showed that CD68 transcript was up-regulated about 3.59-fold in BDL-water treatment group compared with sham group $(P<0.01)$. CD68 mRNA expression was reduced in BDL-XYXD kidneys $(P<0.05)$ indicating that $X Y X D$ could inhibit macrophage activation or promote its apoptosis.

Since pro-inflammatory cytokines are mainly derived from macrophages after renal injury, we examined various pro-inflammatory cytokines and chemokine expression. As presented in Figure $3 C$ and D, mRNA expression levels of CCL2 and TNF- $\alpha$ increased significantly $(P<0.01)$ to more than 5.56- and 4.84-fold, respectively, in BDL-treated kidneys compared with sham as determined by real-time PCR analysis. This increase of pro-inflammatory cytokine expression was attenuated in BDL-XYXD kidneys compared BDL-water kidneys.
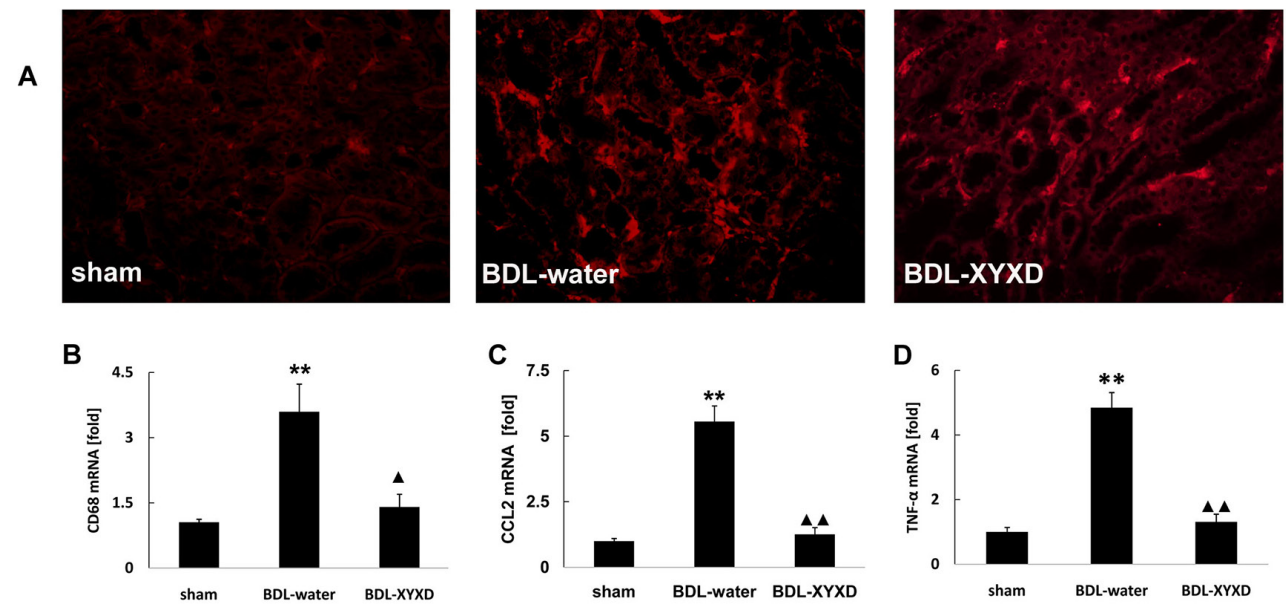

Figure 3. Effects of XYXD on renal macrophages and pro-inflammatory cytokines in rats with cirrhosis. A. CD68 detected by immunofluorescence (400X, N = 4); CD68 (B), CCL2 (C), and TNF- $\alpha$ (D) were measured by real-time $\mathrm{PCR}(\mathrm{N}=6)$. Data are reported as means $\pm \mathrm{SD}$. ${ }^{*} \mathrm{P}<0.05$, ${ }^{*} \mathrm{P}<0.01$, vs sham; ${ }^{\Delta} \mathrm{P}<0.05,{ }^{\Delta} \mathrm{P}<0.01$ vs $\mathrm{BDL}$-water.

\section{XYXD induces renal macrophage apoptosis}

As shown in Figure 3, XYXD decreased CD68 expression, so we investigated if XYXD induced macrophage apoptosis. We first detected apoptotic-related factors such as Fas and Bax expression in BDL-treated kidneys. The expression of Fas and Bax was found to be significantly increased following 5 weeks of BDL treatment (Figure 4A). XYXD administration did not result in significant reductions in Fas and Bax expression compared with BDL-water kidney, which indicated that the apoptotic cell number was not significantly different between BDL-water and BDL-XYXD groups. Further, using double staining, we revealed that XYXD promoted macrophages apoptosis in kidneys.

TUNEL-positive cells in the kidney increased after 5 weeks of BDL-treatment (Figure 4B). There was no difference in the number of TUNEL-positive cells between BDL-water and BDLXYXD kidneys. Apoptotic macrophages shown as double-positive for TUNEL and CD68 were rarely observed in the sham. Double CD68+/TUNEL+ cells also were relatively in low BDL-water group. The number of cells co-staining for TUNEL and CD68 (arrow) were observed in BDL-XYXD 
rat kidneys.

These results showed that (1) renal cell underwent apoptosis during BDL-induced liver cirrhosis; (2) XYXD promoted renal macrophage apoptosis.

Fas

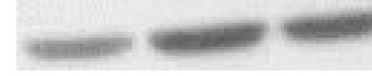

A

Bax

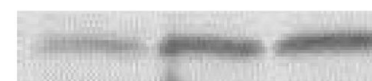

GAPDH

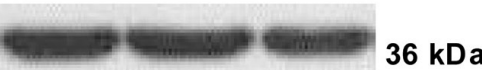

Sham BDL-water BDL-XYXD

\section{$45 \mathrm{kDa}$}

$21 \mathrm{kDa}$

$36 \mathrm{kDa}$
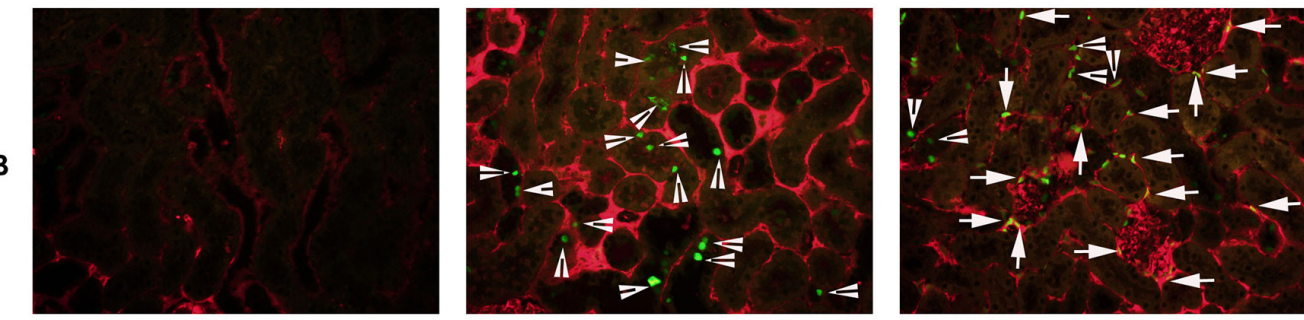

Figure 4. Renal apoptosis factors and macrophage apoptosis were detected using immunofluorescence microscopy in rats. A. Fas and Bax detected by western blotting $(\mathrm{N}=6)$. B. Renal paraffin sections stained with TUNEL (green) and CD68 (red) (400X, N = 4). Arrow points to CD68+ macrophage apoptosis; the arrowhead indicates non-CD68+ macrophage apoptosis.

\section{Levels of pro-fibrotic factors increased in the kidneys of BDL-induced cirrhotic rats}

Renal chronic interstitial inflammation could promote the increase of renal pro-fibrotic factors. Therefore, we detected Col 1 production in the injured kidneys. The results showed that sparse amount of Col 1 was present in the peritubular and glomerular basement membrane in sham kidneys (Figure 5A). After 5-week BDL treatment, Col 1 increased markedly with strong staining, not only located in peritubular and glomerular areas, but also the tubular epithelial cells in BDL-water kidneys. These results indicated chronic renal injury caused by Col production, mainly derived from fibroblasts. Therefore, using antibody against $\alpha$-SMA, a marker of fibroblasts, we assayed the expression of this protein in immunohistochemical analysis (Figure 5B). In sham rats, vascular smooth muscle cells were weakly positive for $\alpha$-SMA and $\alpha$-SMA-positive fibroblasts increased after 5-week BDL treatment. After treatment with XYXD, the increased expression of $\alpha-S M A-p o s i t i v e$ fibroblasts was reduced. We detected $\alpha$-SMA by real-time PCR (Figure 5C). Levels of a-SMA mRNA increased significantly in the BDL-water kidney compared with that in sham group $(P<0.01)$. In the BDL-XYXD group, a-SMA mRNA expression decreased remarkably $(P<0.01)$ compared with the BDL-water group.

As shown in Figure $5 \mathrm{D}$ and $E$, the expression of TGF- $\beta$ and $p-S m a d 2 / 3$ increased 
significantly after 5-week BDL treatment and these trends were almost identical with that of $\alpha$-SMA. Compared to BDL-water kidneys, XYXD administration resulted in marked reductions in TGF- $\beta$ and $\mathrm{p}-\mathrm{Smad} 2 / 3$.

Taken together, these results confirmed that BDL administration caused renal fibroblast activation and increased production and accumulation of pro-fibrotic factors, which might have facilitated or resulted in renal injury in BDL-treated rats. XYXD remarkably reduced pro-fibrotic factors production in the kidneys compared with BDL-water group.
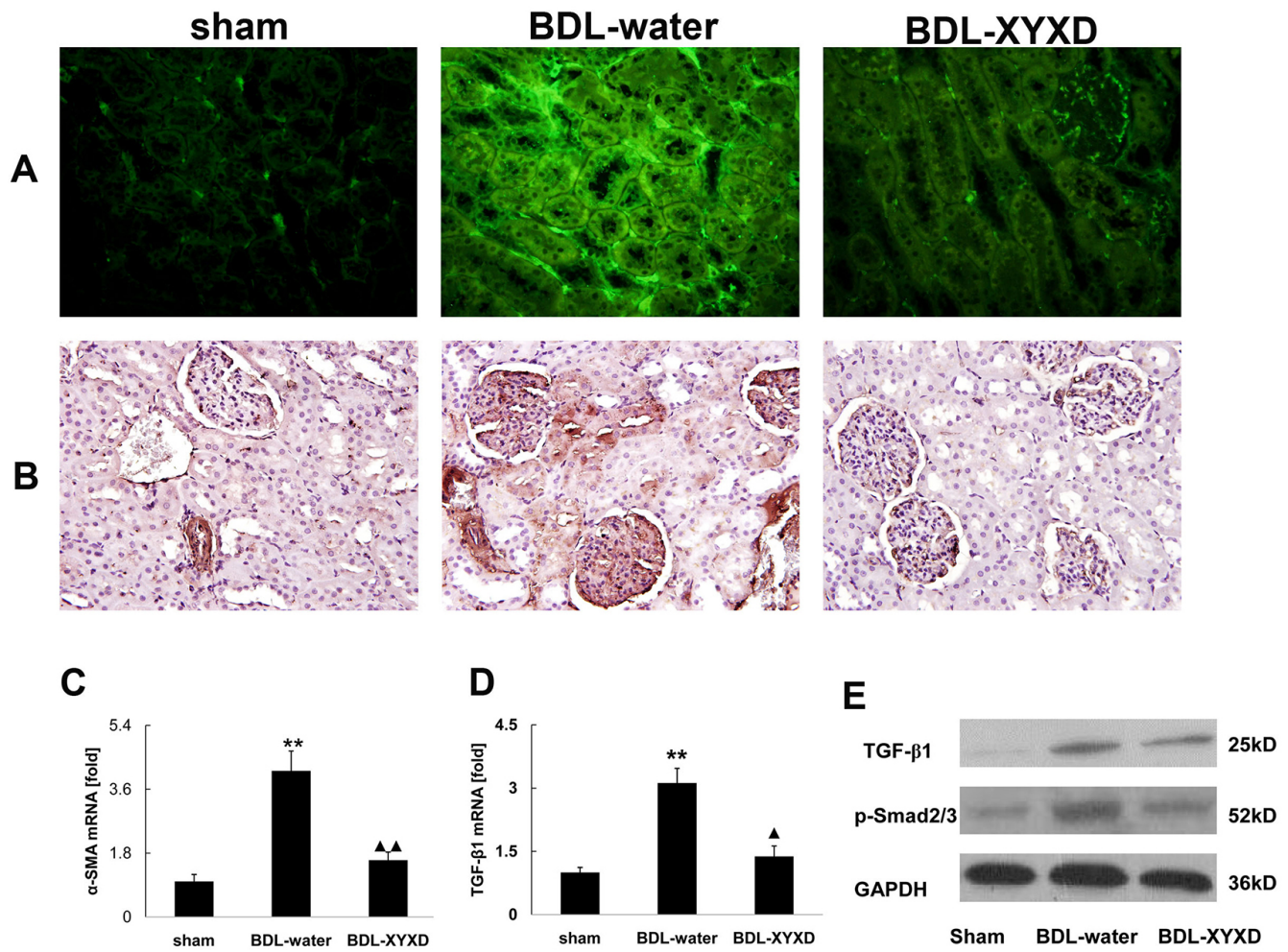

Figure 5. Effects of XYXD on pro-fibrogenic factors in kidneys in BDL-induced cirrhosis. A. Kidney sections stained with Col 1 (Green) (400X, N = 4). B. Kidney sections stained with $\alpha-S M A$ antibody. Brown staining indicates immunopositivity (400X, N = 4); expression of $\alpha-S M A$ (C) and TGF- $\beta 1$ (D) was analyzed using real-time PCR; E. expression of TGF- $\beta 1$ and $\mathrm{p}-\mathrm{Smad} 2 / 3$ analyzed using western blot $(\mathrm{N}=6)$.

\section{XYXD-mediated inhibition of interaction between macrophages and $\alpha-S M A+$ cells}

The pathophysiological involvement of macrophages in the kidney was elucidated by colocalization of CD68 and a-SMA immunofluorescence. CD68 macrophages were observed near or directly adhered to a-SMA+ cells, while both cell types were not observed in the sham kidney (Figure 6). XYXD was found to reduce a-SMA and CD68 expression. These results collectively demonstrated that the protective role of $X Y X D$ on the kidney resulted from induction of macrophage apoptosis. The more the interaction between macrophage and $\alpha-S M A+$ cells was inhibited, further 

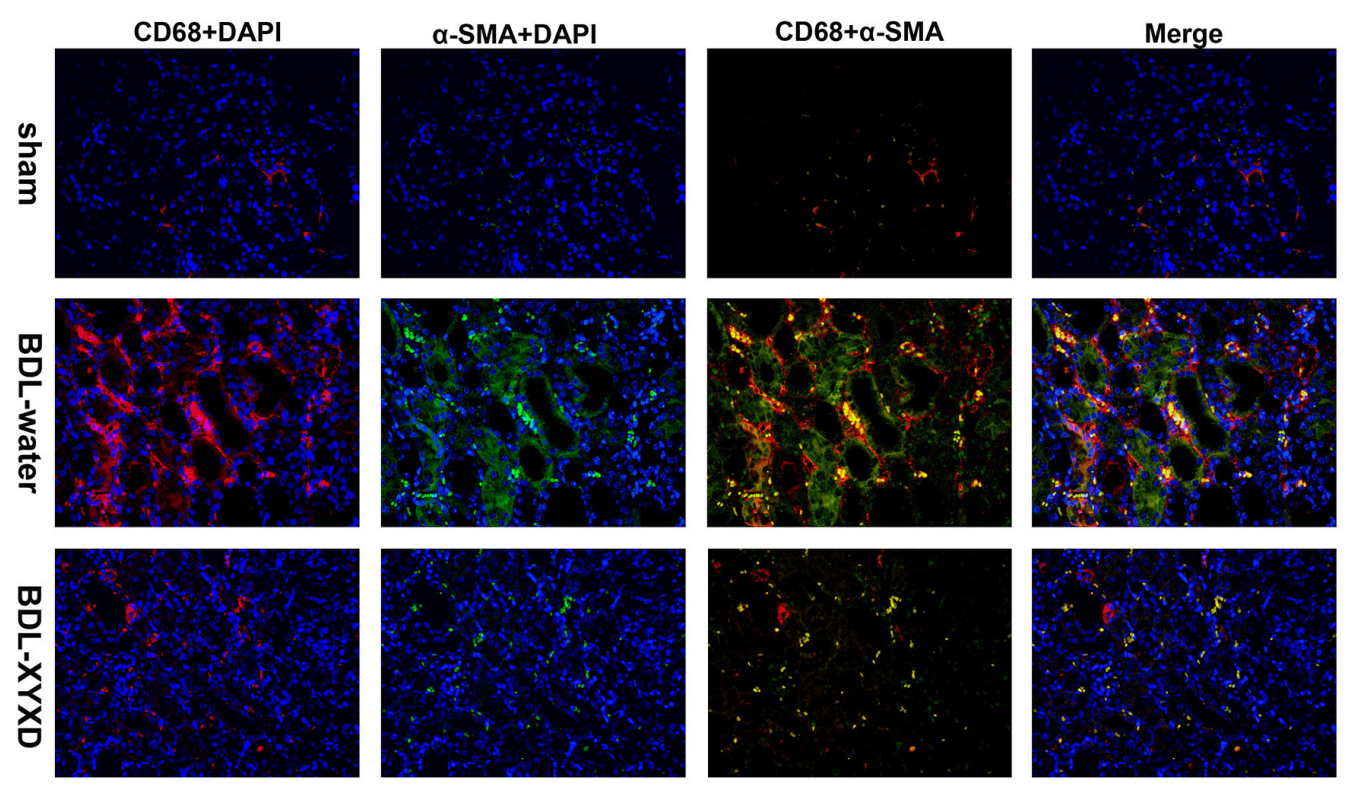

Figure 6. Effects of XYXD on interaction of macrophage and a-SMA+ cells in kidneys of cirrhotic rats. Renal sections stained with $\alpha$-SMA (green), CD68 (red), and DAPI (blue). The yellow color indicates co-localization of CD68 macrophages and $\alpha-S M A+$ cells. (Original magnification 400X, $N=4$ ). the injury reduced.

\section{DISCUSSION}

Hepatorenal syndrome is common in patients with end-stage cirrhosis (Li et al., 2012; Arroyo, 2013; Belcher et al., 2014). However, renal pathology during liver cirrhosis has not been well documented (Natarajan et al., 2006). This study demonstrates that the kidney is damaged and renal macrophage activation is disrupted in rats with liver cirrhosis. XYXD administration attenuates renal injury at least in part, by promoting macrophage apoptosis.

There is overwhelming evidence that the kidneys and renal function are impaired in patients with cirrhosis (Natarajan et al., 2006; Belcher et al., 2014). Our results showed that the kidney weight, ratio of kidney weight/body weight, and renal function changed remarkably in cirrhotic rats. Additionally, we observed that glomerulus diameter and inflammatory cell infiltration in the kidneys increased significantly. All these data indicate renal damage in rats with cholestatic cirrhosis. This result was consistent with those of previous reports that kidneys are damaged in CCL4- and TAAinduced liver cirrhosis due to oxidative stress (Natarajan et al., 2006). Although CCL4 and TAA are toxic to other organs as well, these results suggest that liver cirrhosis is a systemic phenomenon, probably encompassing all tissues and organs.

Macrophages are known to play an important role in liver fibrosis and cirrhosis (Heinrichs et al., 2011; Zimmermann et al., 2011; Heymann et al., 2012; Ramachandran et al., 2012), which is a central component of the final, common pathway leading to HSC activation and ECM deposition (Liu et al., 2008, 2010, 2012; Chiu et al., 2014). However, the macrophage in kidneys with cirrhosis has not been well studied. Our results demonstrate that renal macrophages are disrupted during liver cirrhosis. In this study, CD68, a macrophage marker, increased after 5-week BDL treatment. 
The CD68+ macrophages were distributed mainly in the glomeruli, between renal tubules. Proinflammatory cytokines were also increased in the kidneys. These results indicate that macrophages actively participate in renal damage during liver cirrhosis. CD68 immunohistochemical results also illustrate that renal function and macrophage activation was inseparable. The rampant activation of macrophages around the tubular epithelial cells and glomeruli indicates that renal function must be affected and kidneys are damaged.

We previously reported that macrophages underwent apoptosis in dimethylnitrosamineinduced liver fibrosis (Liu et al., 2010). Therefore, in this study, we investigated if macrophages underwent apoptosis in the kidneys during liver cirrhosis. The results showed that almost none of the CD68+ macrophages underwent apoptosis in BDL-water kidneys. However, CD68+ macrophages underwent apoptosis in BDL-XYXD kidneys. Macrophages are known to secrete pro-inflammatory cytokines and pro-fibrotic cytokines in progressive diseases, regardless of the initial cause of injury (Yamate et al., 2004; Heinrichs et al., 2011). In this study, macrophages underwent apoptosis and the quantity of pro-inflammatory cytokines decreased, so renal injury seemed to have reduced in the BDL-XYXD group.

Chronic, progressive renal injury results in renal fibrosis (Chen et al., 2014; Ji et al., 2014). Surprisingly, immunofluorescence and immunohistochemistry results revealed a marked increase in the accumulation of $\mathrm{Col} 1$ and $\alpha$-SMA+ myofibroblasts in the kidneys of the 5-week BDL-watertreated group, as determined by real-time PCR. It has been reported that TGF- $31 /$ Smad signaling is a key mediator of renal tubular interstitial fibrosis (Nishida et al., 2007; Okano et al., 2010; Choi et al., 2011; Falke et al., 2012; Huen et al., 2013). Consistent with these reports, renal fibrosis was also observed in this study. Based on our knowledge, this is the first report of renal fibrosis in hepatic cirrhotic rats.

In summary, this study suggests that renal tissue and function are impaired during development of liver cirrhosis. XYXD reduces renal injury by promoting macrophage apoptosis. These results highlight some important new concepts: the kidney is also damaged during liver cirrhosis and therefore, protection of the kidneys is essential during treatment.

\section{Conflicts of interest}

The authors declare no conflict of interest.

\section{ACKNOWLEDGMENTS}

Research mainly supported in whole or part by the Putuo Hospital (\#2014YJ001, to C. Liu), the Shanghai Municipal Public Health Bureau (\#201440370, to D. Xue), and the Shanghai Putuo Science and Technology Commission Project (\#2011PTKW006, to D. Xue).

\section{REFERENCES}

Andersen ES, Rodgaard-Hansen S, Moessner B, Christensen PB, et al. (2014). Macrophage-related serum biomarkers soluble CD163 (sCD163) and soluble mannose receptor (SMR) to differentiate mild liver fibrosis from cirrhosis in patients with chronic hepatitis C: a pilot study. Eur. J. Clin. Microbiol. Infect. Dis. 33: 117-122.

Arroyo V (2013). Acute kidney injury (AKI) in cirrhosis: should we change current definition and diagnostic criteria of renal failure in cirrhosis? J. Hepatol. 59: 415-417.

Belcher JM, Sanyal AJ, Peixoto AJ, Perazella MA, et al. (2014). Kidney biomarkers and differential diagnosis of patients with 
cirrhosis and acute kidney injury. Hepatology 60: 622-632.

Chen L, Li J, Zhang J, Dai C, et al. (2014). S100A4 promotes liver fibrosis via activation of hepatic stellate cells. J. Hepatol. 62: 156-164.

Chen SL, Du GL, Ding N, Jin HX, et al. (2012). Comparative study of Xiayuxue decoction and its ingredients recipe against porcine serum induced liver fibrosis in rats. Chin. J. Exp. Trad. Med. Formulae 18: 154-157.

Chen XH (2013). Xia-yu-xue decoction treatment on 45 cases of early cirrhosis. Zhengjiang J. TCM 48: 705.

Chiu YS, Wei CC, Lin YJ, Hsu YH, et al. (2014). IL-20 and IL-20R1 antibodies protect against liver fibrosis. Hepatology 60 : 1003-1014.

Choi DE, Jeong JY, Lim BJ, Chang YK, et al. (2011). Aliskiren ameliorates renal inflammation and fibrosis induced by unilateral ureteral obstruction in mice. J. Urol. 186: 694-701.

Ding SD, Liu BC, Liu Y, Dong QT, et al. (2012). Proteomic study of Xiayuxue decoction on liver cirrhosis of rats. Zhong Cao Yao 01: 131-138.

Du YG, Yang MH, Chai KF and Jin GY (2010). Effects of Xinjia Xiayuxue Tang on bone morphogenetic protein 7 and transforming growth factor $\beta 1$ in kidney of diabetic rats. Chin J. Exp. Trad. Med. Formulae 11: 135-138.

Falke LL, Dendooven A, Leeuwis JW, Nguyen TQ, et al. (2012). Hemizygous deletion of CTGF/CCN2 does not suffice to prevent fibrosis of the severely injured kidney. Matrix Biol. 31: 421-431.

Francis H, Franchitto A, Ueno Y, Glaser S, et al. (2007). H3 histamine receptor agonist inhibits biliary growth of BDL rats by downregulation of the cAMP-dependent PKA/ERK1/2/ELK-1 pathway. Lab. Invest. 87: 473-487.

Graf A, Meng F, Hargrove L, Kennedy L, et al. (2014). Knockout of histidine decarboxylase decreases bile duct ligationinduced biliary hyperplasia via downregulation of the histidine decarboxylase/VEGF axis through PKA-ERK1/2 signaling. Am. J. Physiol. Gastrointest. Liver Physiol. 307: G813-G823.

Heinrichs D, Knauel M, Offermanns C, Berres ML, et al. (2011). Macrophage migration inhibitory factor (MIF) exerts antifibrotic effects in experimental liver fibrosis via CD74. Proc. Natl. Acad. Sci. U. S. A. 108: 17444-17449.

Heymann F, Hammerich L, Storch D, Bartneck M, et al. (2012). Hepatic macrophage migration and differentiation critical for liver fibrosis is mediated by the chemokine receptor C-C motif chemokine receptor 8 in mice. Hepatology 55: 898-909.

Hong Y, Shi Y, Liao W, Klahr NJ, et al. (2014). Relative ADC measurement for liver fibrosis diagnosis in chronic hepatitis $\mathrm{B}$ using spleen/renal cortex as the reference organs at $3 \mathrm{~T}$. Clin. Radiol. 69: 581-588.

Huen SC, Moeckel GW and Cantley LG (2013). Macrophage-specific deletion of transforming growth factor-beta1 does not prevent renal fibrosis after severe ischemia-reperfusion or obstructive injury. Am. J. Physiol. Renal. Physiol. 305: F477-F484.

Ji L, Xue R, Tang W, Wu W, et al. (2014). Toll like receptor 2 knock-out attenuates carbon tetrachloride (CCl4)-induced liver fibrosis by downregulating MAPK and NF-KB signaling pathways. FEBS Lett. 588: 2095-2100.

Li P, Zhang HJ and Zheng LT (2012). The theory of homogeny of liver and kidney in the treatment of kidney and liver fibrosis. Chin. J. Integr. Med. 18: 250-252.

Liu C, Sun M, Yan X, Han L, et al. (2008). Inhibition of hepatic stellate cell activation following Yinchenhao decoction administration to dimethylnitrosamine-treated rats. Hepatol. Res. 38: 919-929.

Liu C, Tao Q, Sun M, Wu JZ, et al. (2010) Kupffer cells are associated with apoptosis, inflammation and fibrotic effects in hepatic fibrosis in rats. Lab. Invest. 90: 1805-1816.

Liu C, Wang G, Chen G, Mu Y, et al. (2012). Huangqi decoction inhibits apoptosis and fibrosis, but promotes Kupffer cell activation in dimethylnitrosamine-induced rat liver fibrosis. BMC Complement. Altern. Med. 12: 51.

Mejias M, Garcia-Pras E, Tiani C, Miquel R, et al. (2009). Beneficial effects of sorafenib on splanchnic, intrahepatic, and portocollateral circulations in portal hypertensive and cirrhotic rats. Hepatology 49: 1245-1256.

Natarajan SK, Basivireddy J, Ramachandran A, Thomas S, et al. (2006). Renal damage in experimentally-induced cirrhosis in rats: Role of oxygen free radicals. Hepatology 43: 1248-1256.

Nishida M, Okumura Y, Ozawa S, Shiraishi I, et al. (2007). MMP-2 inhibition reduces renal macrophage infiltration with increased fibrosis in UUO. Biochem. Biophys. Res. Commun. 354: 133-139.

Okano K, Tsuruta Y, Yamashita T, Takano M, et al. (2010). Suppression of renal fibrosis by galectin-1 in high glucosetreated renal epithelial cells. Exp. Cell Res. 316: 3282-3291.

Patsenker E, Popov Y, Stickel F, Schneider V, et al. (2009). Pharmacological inhibition of integrin alphavbeta3 aggravates experimental liver fibrosis and suppresses hepatic angiogenesis. Hepatology 50: 1501-1511.

Ramachandran P, Pellicoro A, Vernon MA, Boulter L, et al. (2012). Differential Ly-6C expression identifies the recruited macrophage phenotype, which orchestrates the regression of murine liver fibrosis. Proc. Natl. Acad. Sci. U. S. A. 109: E3186-E3195.

Wong F, Moore K, Dingemanse J and Jalan R (2008). Lack of renal improvement with nonselective endothelin 
antagonism with tezosentan in type 2 hepatorenal syndrome. Hepatology 47: 160-168.

Yamate J, Machida Y, Ide M, Kuwamura M, et al. (2004). Effects of lipopolysaccharide on the appearance of macrophage populations and fibrogenesis in cisplatin-induced rat renal injury. Exp. Toxicol. Pathol. 56: 13-24.

Zhang LJ, Sun MY, Ning BB, Zhang WM, et al. (2014). Xiayuxue Decoction ([symbols; see text]) attenuates hepatic stellate cell activation and sinusoidal endothelium defenestration in CCl4-induced fibrotic liver of mice. Chin. J. Integr. Med. 20: 516-523.

Zimmermann HW, Seidler S, Gassler N, Nattermann J, et al. (2011). Interleukin-8 is activated in patients with chronic liver diseases and associated with hepatic macrophage accumulation in human liver fibrosis. PLoS One 6: e21381. 